СТАТЬИ

УДК 519.6

РЕШЕНИЕ ЗАДАЧИ ОПТИМАЛЬНОГО УПРАВЛЕНИЯ
ВЕРТИКАЛЬНЫМ ПОДЬЕМОМ РАКЕТЫ-ЗОНДА С УТОЧНЕНИЕМ
МОДЕЛИ АЭРОДИНАМИЧЕСКОГО СОПРОТИВЛЕНИЯ

Мозжорина Т.Ю., Попов А.С.

Московский государственный технический университет имени Н.Э. Баумана (национальный исследовательский университет), Москва, e-mail: mozzhorina@mail.ru

В данной работе решается задача оптимального управления метеорологической ракетой-зондом при ее вертикальном подъеме с уточнением математической модели аэродинамического сопротивления, что отличает ее от предшествующих исследований, посвященных этому вопросу. Оптимизация основана на применении принципа максимума Понтрягина и численных методов. Качественное решение данной задачи оптимального управления известно: вначале тяга должна быть максимально возможной, в дальнейшем, начиная с какого-то момента времени, следует ее уменьшить. Управление на участке пониженной тяги является здесь особым режимом управления. Проведен численный эксперимент, показавший, что наличие особого режима при оптимальном управлении связано с аэродинамическим сопротивлением. Осуществлена попытка решения краевой задачи оптимального управления с переключением и с наличием особого режима управления методом пристрелки. В качестве исходных данных взяты технические характеристики ракеты МР-20. В настоящее время особый режим управления для метеорологических ракет вертикального взлета не применяется в силу необходимости использования регулируемого твердотопливного двигателя, создание которого представляет собой некоторые технические сложности. В силу этого представляет интерес возможность количественной оценки преимуществ, которые может дать оптимальное управление тягой двигателя при наличии особого режима управления. Проводится численный анализ эффективности применения особого режима управления.

Ключевые слова: оптимальное управление, принцип максимума Понтрягина, режим особого управления, метод пристрелки решения краевых задач, метеорологическая ракета-зонд

\title{
SOLUTION OF THE PROBLEM OF OPTIMUM CONTROL OF THE VERTICAL LIFT OF THE PROBE ROCKET WITH REFINING THE AERODYNAMIC RESISTANCE MODEL
}

Mozzhorina T.Yu., Popov A.C.

Bauman Moscow State Technical University, Moscow, e-mail: mozzhorina@mail.ru

This paper solves the problem of optimal control of a meteorological probe rocket during its vertical ascent with the refinement of the mathematical model of aerodynamic drag, which distinguishes it from previous studies devoted to this issue. Optimization is based on the application of the Pontryagin maximum principle and numerical methods. The qualitative solution of this problem of the optimal control is known: at first, the thrust should be as high as possible, then, starting from a certain point in time, it should be reduced. Low thrust control is a special control mode here. A numerical experiment has been carried out, which showed that the presence of a special regime under optimal control is associated with aerodynamic drag. An attempt is made to solve the boundary value problem of optimal control with switching and with the presence of a special control mode by the shooting method. The technical characteristics of the MR-20 rocket were taken as the initial data. Currently, the special control mode for vertical take-off meteorological rockets is not applied due to the need to use a regulated solid-propellant engine, the creation of which presents some technical difficulties. Because of this, it is of interest to be able to quantify the benefits that can be obtained from optimal engine thrust control in the presence of a special control mode. A numerical analysis of the effectiveness of the use of a special control mode is carried out.

Keywords: optimal control, Pontryagin's maximum principle, special control mode, shooting method for solving boundary value problems, meteorological probe rocket

Эта классическая задача оптимального управления ранее была неоднократно решена и результаты представлены в таких работах, как $[1,2]$. Следует, однако, отметить, что для аэродинамического сопротивления в них были использованы упрощенные зависимости. В [1] полуэмпирическая формула для аэродинамического сопротивления

$$
\mathrm{X}_{\text {аэр }}=\frac{c_{x} \rho V^{2} S}{2},
$$

где $c_{x}-$ коэффициент аэродинамического сопротивления, $\rho$ - плотность воздуха, зависящая от высоты, $V$ - скорость полета,
$S$ - характерная площадь летательного аппарата (в данной задаче площадь миделя ракеты) применялась с допущениями: $c_{x}=$ const, плотность воздуха аппроксимировалась экспоненциальной зависимостью по высоте $\rho=a_{1} \cdot e^{-a_{2} h}$. Положительные коэффициенты $a_{1}, a_{2}$, вероятно, были подобраны методом наименьших квадратов. Из экспериментальных данных известно, что коэффициент аэродинамического сопротивления сильно зависит от числа Маха полета (рис. 1 из [3]), а кривая плотности, рассчитанная в соответствии с [4], не очень точно аппроксимируется экспоненциальной зависимостью (рис. 2). 


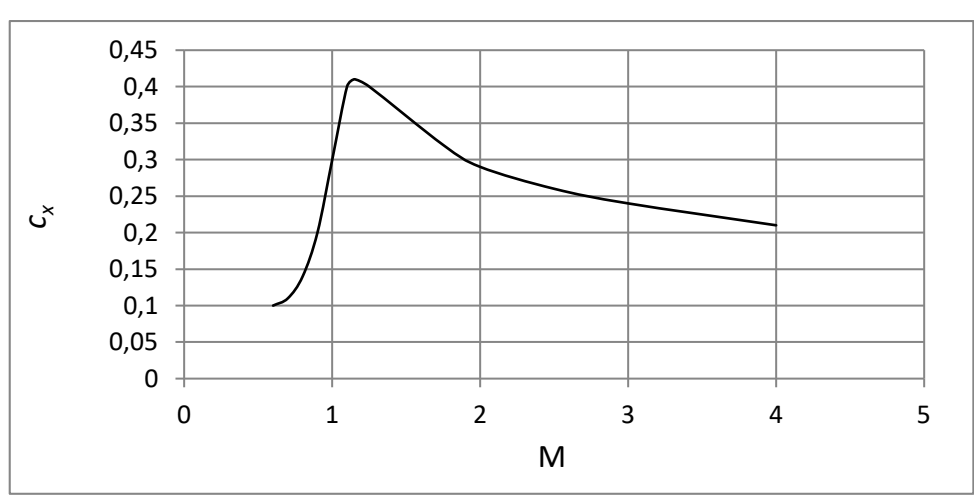

Рис. 1. Коэффициент аэродинамического сопротивления ракеть при угле атаки равном нулю в зависимости от числа Маха

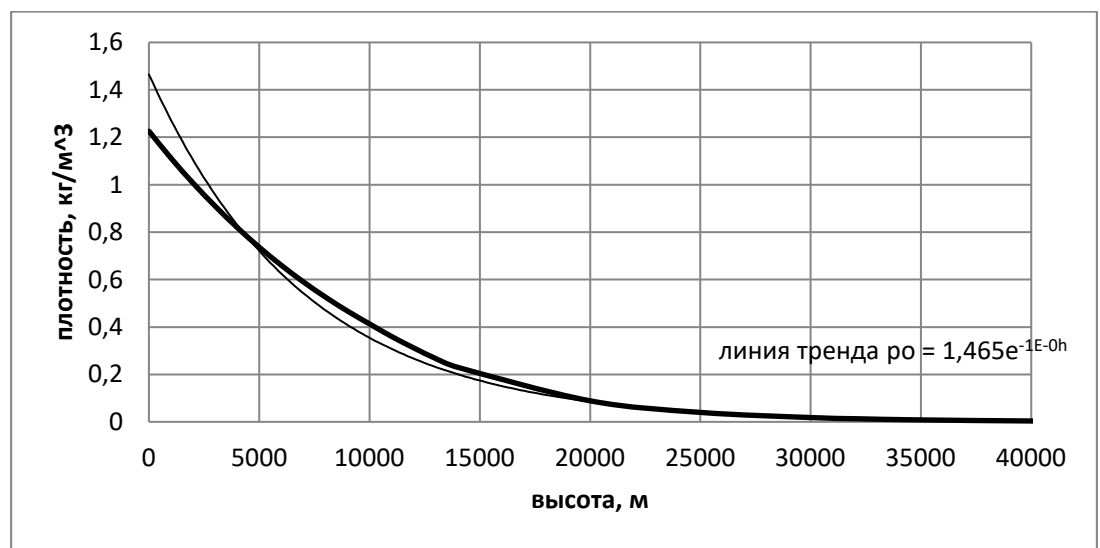

Рис. 2. Аппроксимаџия плотности воздуха по высоте экспоненциильной зависимостью

В работе [2] также принималось постоянство коэффициента аэродинамического сопротивления.

Качественное решение задачи ОУ выглядит следующим образом: в начале взлета двигатель должен работать на максимальном режиме, далее следует участок особого режима управления с пониженной тягой, по окончанию топлива - движение по инерции до некоторой высоты.

Цель исследования: провести численный эксперимент для метеорологической ракеты типа MP20 с уточнением математической модели для аэродинамического сопротивления в соответствии с [3] и [4], по полученным количественным результатам расчетов провести анализ целесообразности применения особого режима управления.

\section{Математические модели и численные методы}

При заданном весовом балансе ракеты будем максимизировать высоту подъема ракеты.

$$
J=h_{\text {подъема }} \rightarrow \max \text { или }
$$

интегральный критерий $J=-\int_{0}^{T} V d t \rightarrow \min$

Система уравнений движения при вертикальном подъеме ракеты-зонда:

$$
\left\{\begin{array}{l}
\frac{d h}{d t}=V \\
\frac{d V}{d t}=\frac{\beta u-X_{a \ni p}}{m}-g \\
\frac{d m}{d t}=-G_{T}=-u
\end{array}\right.
$$

где $h$ - высота, м; $V$ - скорость, м/с; $P=\beta u$ тяга двигателя, $\mathrm{H} ; \beta$ - импульс РДТТ, м/с; $u=G_{T}-$ управление, расход топлива, кг/с; $X_{\text {аэp }}$ - сила аэродинамического сопротивления, $\mathrm{H} ; m$ - масса ракеты, кг; $t$ - время, с; $g=9,81 \frac{R^{2}}{(R+h)^{2}}-$ ускорение свободного падения, м/ $\mathrm{c}^{2}, R$ - радиус Земли. В качестве 
исходных данных были приняты технические характеристики метеорологической ракеты МР-20: калибр $d_{\max }=450$ мм, взлетная масса $m_{0}=1620$ кг, импульс двигателя $\beta=2011 \mathrm{м} /$ сек считался постоянным, максимальный расход топлива $u=G_{T}=50,54$ кг/сек, масса топлива 1200 кг.

При использовании принципа максимума Понтрягина функция Понтрягина имеет вид

$H=\psi_{h} V+\psi_{V}\left(\frac{\beta u-X_{a э p}}{m}-g\right)+\psi_{m}(-u)+V=$ $=\psi_{h} V+\psi_{V}\left(-\frac{X_{a \ni p}}{m}-g\right)+V+u\left(\psi_{V} \frac{\beta u}{m}-\psi_{m}\right)$.

$\psi_{h}, \psi_{V}, \psi_{m}$ - сопряженные переменные.

Функция линейна по управлению, коэффициент при управлении - функция переключения

$$
\Phi_{n e p^{\prime}}=\psi_{V} \frac{\beta u}{m}-\psi_{m} .
$$

Оптимальное управление в этом случае $u^{*}=\left\{\begin{array}{l}u_{\max } \quad \text { при } \Phi_{n е р}>0 \\ 0 \quad \text { при } \Phi_{\text {nep }}<0 \\ u \in\left[0, u_{\max }\right] \text { при } \Phi_{\text {nep }}=0 \quad \forall t \in\left[t_{1}, t_{2}\right] \subset[0, T]\end{array}\right.$

Последний случай относится как раз к особому режиму управления. Исследованиям управления с особым режимом посвящены такие работы, как $[5,6,7]$. В этом случае должно выполняться на $\left[t_{1}, t_{2}\right]$

$$
\frac{d \Phi}{d t}=0, \frac{d^{2} \Phi}{d t^{2}}=0 .
$$

Вначале был рассмотрен случай без особого режима управления, решение краевой задачи для системы ДУ

$$
\left\{\begin{array}{l}
\frac{d h}{d t}=V \\
\frac{d V}{d t}=\frac{\beta u^{*}-X_{a \ni p}}{m}-g \\
\frac{d m}{d t}=-G_{T}=-u^{*} \\
\frac{d \psi_{h}}{d t}=-\frac{\partial H}{\partial h} \\
\frac{d \psi_{V}}{d t}=-\frac{\partial H}{\partial V} \\
\frac{d \psi_{m}}{d t}=-\frac{\partial H}{\partial m}
\end{array}\right.
$$

с краевыми условиями:

$$
\begin{gathered}
h(0)=0, V(0)=0, m(0)=m_{0}=1620 \kappa \Gamma, \\
V(T)=0, m(T)=m_{0}-m_{\text {топл }}=420 \kappa \Gamma, \psi_{h}(T)=0
\end{gathered}
$$

было получено методом пристрелки. Метод пристрелки или стрельбы дает наиболее точные результаты численного решения краевых задач $[1,8]$. Производные функции Понтрягина по сопряженным переменным вычислялись с учетом того, что плотность воздуха, скорость звука и, соответственно, число Маха полета, а следовательно, и коэффициент аэродинамического сопротивления являются функциями высоты. Коэффициент аэродинамического сопротивления является также еще и функцией скорости полета. Внешний цикл - модифицированный метод Ньютона обращался к численному методу решения задачи Коши - методу Рунге - Кутты 4-го порядка. Для сходимости метода Ньютона момент отключения двигателя по израсходованию запаса топлива уточнялся линейной интерполяцией массы ракеты на шаге метода Рунге - Кутты, на котором заканчивалось топливо. После чего этот шаг пересчитывался с тягой двигателя, равной среднеинтегральному значению по указанному промежутку времени. Алгоритм пересчета был предложен для задач оптимального управления с переключением в [1], также применяя этот алгоритм, удалось достичь сходимости метода Ньютона в задачах с переключением в [9] и [10].

Параметры пристрелки и невязки для этого варианта расчета выбирались следующие:

$$
\begin{gathered}
\psi_{h}(0), \psi_{V}(0), \psi_{m}(0), \mathrm{T}, \\
\delta_{1}=V(T) \rightarrow 0, \delta_{2}=\psi_{h}(T) \rightarrow 0, \\
\delta_{3}=H(T) \rightarrow 0, \delta_{4}=\Phi_{n e p}\left(t_{n e p}\right) \rightarrow 0 .
\end{gathered}
$$

Полученные результаты не соответствовали теории: функция Понтрягина не была равна нулю на всем протяжении, функция переключения меняла знак дважды, причем в точке переключения меняла знак с минуса на плюс, а не наоборот. Таким образом, вывод о неоптимальности управления без особого режима подтвердился. При этом при обнулении силы аэродинамического сопротивления в математической модели функция переключения и функция Понтрягина вели себя согласно теории: функция Понтрягина была равна нулю с точностью до 4-го знака, функция переключения меняла знак один раз с плюса на минус именно в момент отключения двигателя в связи с израсходованием всего топлива. 
Попытка реализовать алгоритм метода пристрелки для варианта с особым режимом управления при получении оптимального управления на особом режиме из равенства $\frac{d^{2} \Phi}{d t^{2}}=0$ (полное выражение слишком громоздко и в силу этого не приводится) не увенчалась успехом. Оптимальное управление на особом режиме зависит от сопряженных переменных, которые являются параметрами пристрелки в начальный момент времени, и в процессе поиска решения методом Ньютона при неточно выбранном начальном приближении получаемые значения управления на особом режиме не удовлетворяют ограничениям: $u \in\left[0, u_{\max }\right]$.

В силу указанных причин было принято решение отказаться от решения задачи методом пристрелки и реализовать более простой алгоритм с использованием результатов, полученных в предыдущих работах [1] (рис. 3), а именно, постоянства тяги на особом режиме управления.

Алгоритм расчета с двумя переключениями состоял в следующем: первый вызов метода Рунге-Кутты при максимальном значении тяги ограничивался временем $t$, далее осуществлялся второй вызов метода Рунге-Кутты с пониженной тягой двигателя $u=\bar{u}_{\text {otn }} \cdot u_{\text {max }}$, третий вызов при отключенном двигателе ограничивался достижением нулевой скорости полета. Были рассчитаны варианты при значениях $t_{1}=3,5,7,9,11$ секунд. Для каждого из этих вариантов времени также варьировалась величина коэффициента уменьшения тяги $\bar{u}_{\text {otn }}=0,35$; 0,$45 ; 0,55 ; 0,65 ; 0,75,0,85$.

Таким образом, было получено решение для 30 вариантов управления тягой (+1 расчет без особого режима с временем работы двигателя около 24 с).

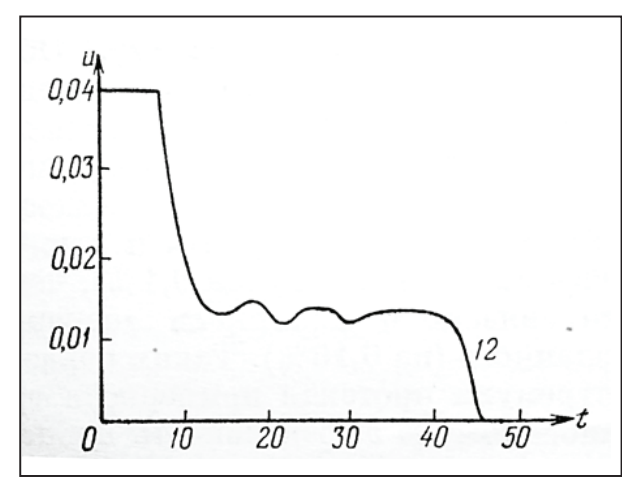

Рис. 3. 12-я итерация решения задачи о вертикальном подъеме ракеть-зонда методом проекиии градиента [1]

\section{Результаты расчетов}

Результаты численного эксперимента для оптимального управления с участком особого режима представлены на рис. 4 и 5.

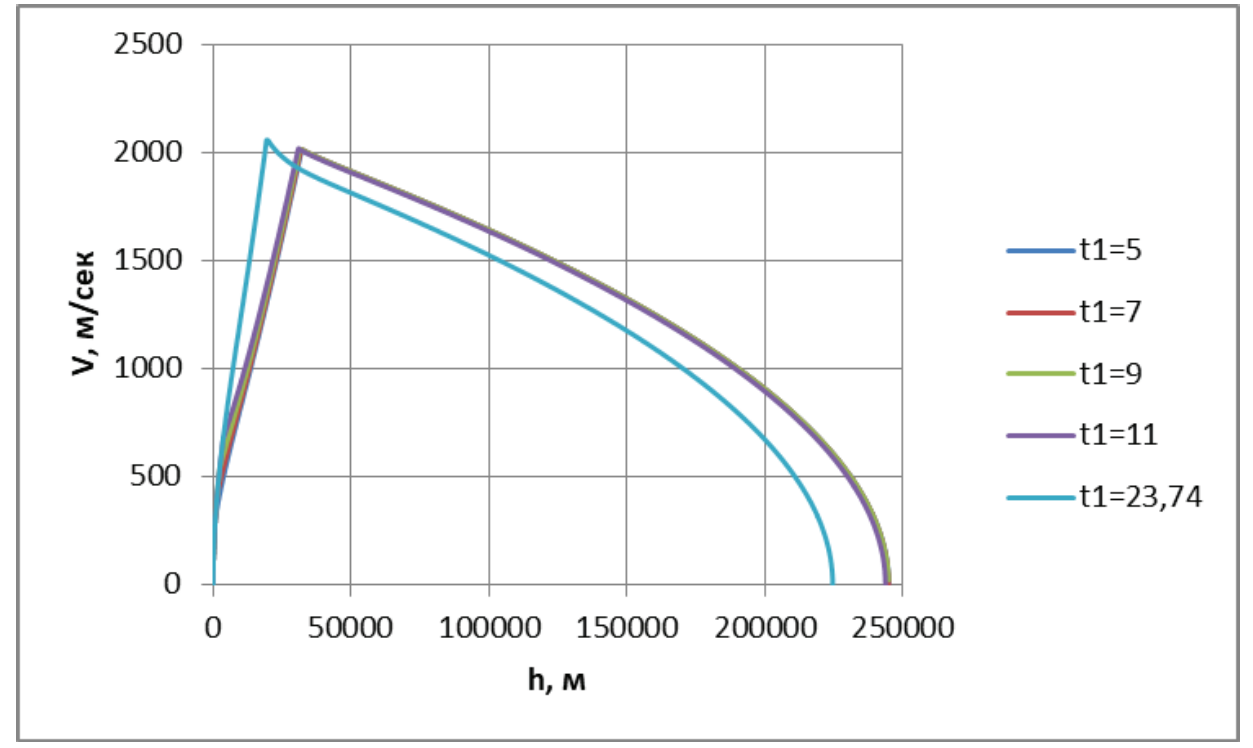

Рис. 4. Изменение скорости по высоте для $\bar{u}_{\text {otn }}=0,55$ при разных значениях времени работы двигателя на максимальном режиме $t_{1}$.

Значение $t_{1}=23,74$ с соответствует варианту без особого режима 


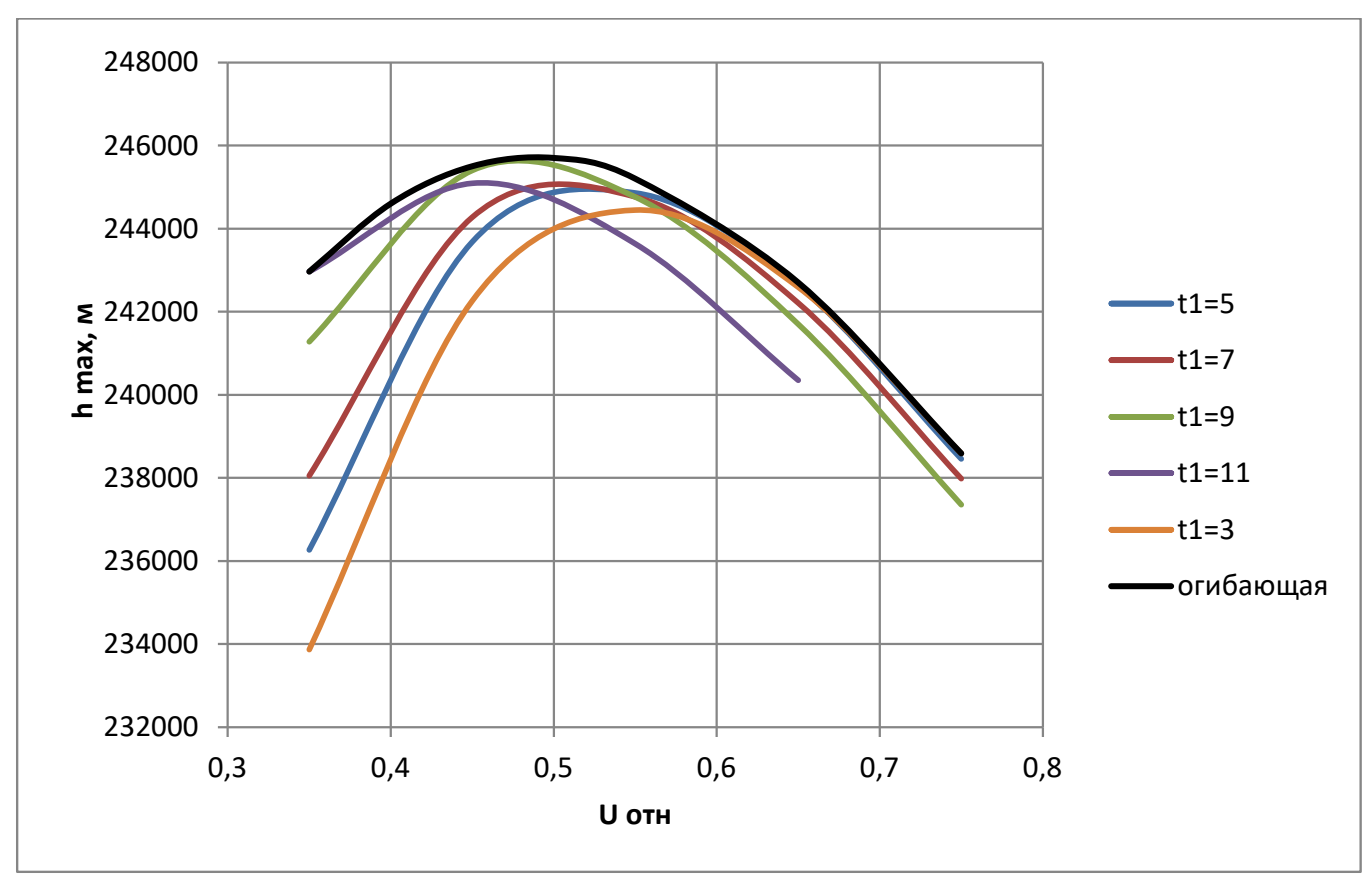

Рис. 5. Достижимая высота при различных вариантах регулирования двигателя

\section{Заключение}

Наличие особого режима управления связано с аэродинамическим сопротивлением воздуха, слишком быстрый набор скорости в плотных слоях атмосферы приводит к большим энергетическим потерям.

Численный метод пристрелки в промежуточных приближениях не дает возможности определения тяги на особом режиме в заданном диапазоне, что приводит к выводу о нецелесообразности применения этого метода для решения задач с переключением и наличием участка особого управления.

При работе на максимальном режиме около 8 с и дальнейшем уменьшении тяги двигателя до $50 \%$ от максимального значения можно увеличить высоту подъема данной ракеты на 25 км, что составляет около $11 \%$ от высоты, достигаемой без применения особого режима управления. Учитывая эти результаты, можно сделать вывод о незначительном увеличении эффективности с применением особого управления при решении подобных задач. С учетом необходимости создания твердотопливного реактивного двигателя с регулируемой тягой для реализации управления с особым режимом целесообразность применения особого режима становится спорной.

\section{Список литературы}

1. Федоренко Р.П. Приближенное решение задач оптимального управления. М: Наука, 1978. 486 с.

2. Данг Нгок Тхань. Разработка вариационного метода и его компьютерного алгоритма оптимизации проектных решений геофизической исследовательской ракеты: автореферат дис. ... канд. техн. наук: 05.07.02 Моск. гос. авиац. ин-т. Москва, 2005. 27 с

3. Пегов В.И., Киселев В.И. Аналитическое представление аэродинамических характеристик летательных аппаратов сложной формы // Наука ЮУрГУ: материалы 66-й научной конференции секции технических наук. 2014. С. 1728-1739.

4. ГОСТ 4401-81 Группа Т27 межгосударственный стандарт атмосфера стандартная. М.: Стандартгиз, 1949.

5. Молоденков А.В., Сапунков Я.Г. Особые режимы управления в задаче оптимального разворота космического аппарата и их приложения // Вестник Нижегородского университета им. Н.И. Лобачевского. Общая и прикладная механика. 2011. № 4 (2). С. 237-238.

6. Хорошавин В.С., Зотов А.В. Особое оптимальное управление нелинейными объектами. Киров: Вятский государственный университет, 2019. 208 с.

7. Хорошавин В.С., Грудинин В.С. Оптимальное программное движение с изменяемым временем регулирования // Радиопромышленность. 2020. Т. 30. № 3. С. 40-49. DOI: $10.21778 / 2413-9599-2020-30-3-40-49$.

8. Афанасьева М.Н., Кузнецов Е.Б. Численный метод решения нелинейной краевой задачи для дифференциальных уравнений с запаздывающим аргументом // Труды МАИ. Выпуск № 88. Математическое моделирование, численные методы и комплексы программ, 2016.

9. Мозжорина Т.Ю. Численное решение задач оптимального управления с переключением методом пристрелки // Математическое моделирование и численные методы. 2017. № 2 (14). С. 94-106.

10. Мозжорина Т.Ю., Осипов В.В. Численное решение задачи о мягком приземлении методом пристрелки // Инновационное развитие. 2018. № 8 (25). С. 11-15. 